\title{
CINÉTICA DE POLIMERIZAÇÃO DE COMPÓSITOS EXPERIMENTAIS BIOATIVOS PARA A COLAGEM ORTODÔNTICA
}

\author{
POLYMERIZATION KINETICS OF EXPERIMENTAL BIOACTIVE COMPOSITES FOR ORTHODONTIC \\ BONDING
}

\section{CINÉTICA DE POLÍMERIZACIÓN DE COMPOSITOS EXPERIMENTALES BIOACTIVOS PARA CEMENTACIÓN DE APARATOLOGÍA ORTODÓNTICA}

\author{
Vinícius Tadeu Pereira Rodrigues ${ }^{1}$, Larissa Pereira Nunes ${ }^{2}$, Rogéria Christina de Oliveira Aguiar ${ }^{3}$, Mary Carmen \\ Treviño Vega ${ }^{4}$, Hélio Doyle Pereira da Silva ${ }^{5}$, Marcela Charantola Rodrigues ${ }^{6}$, Roberto Ruggiero Braga ${ }^{7}$, \\ Marina Guimarães Roscoe ${ }^{8}$
}

\begin{abstract}
RESUMO
Objetivo: Investigar a influência da adição de partículas de fosfato de cálcio na cinética de polimerização e no grau de conversão (GC) de compósitos. Método: Quatro compósitos experimentais foram avaliados: G1- Apenas matriz orgânica à base de metacrilato (MM), G2- Adição de partículas de vidro de bário $(2 \mu \mathrm{m})$ à MM, G3- Adição de nanopartículas de di-hidrato de fosfato dicálcico(DCPD) não funcionalizadas à MM,G4- Adição denanopartículas de DPCD funcionalizadas à MM.O conteúdo de carga inorgânica foi $20 \%$ em volume. O GC foi avaliado por Espectroscopia Infravermelho com Transformada de Fourier. Resultados: Houve aumento do GC para todos os grupos em função do tempo,sem diferença estatística até $5 \mathrm{~s}$.A partir de 11s, o grupo DCPD puro apresentou GC inferior aos demais grupos $(p<0,05)$. Este comportamento manteve-se constante até o final da avaliação. Conclusão: A funcionalização das nanopartículas de DCPD aumentou estatisticamente o GC do material.
\end{abstract}

DESCRITORES: Compósitos experimentais; Grau de conversão; Materiais ortodônticos.

\section{ABSTRACT}

Objective: To investigate the influence of the addition of calcium phosphate particles on the polymerization kinetics and degree of conversion (DC) of experimental composites. Method: Four experimental composites were evaluated. : G1- Only methacrylate-based organic matrix (MM), G2- Addition of barium glass particles (2 $\mu \mathrm{m})$ to the MM, G3-Addition of dicalcium phosphate dihydrate (DCPD) nanoparticles non-functionalized, G4- Addition of DPCD nanoparticles functionalized with TEDGMA. The inorganic filler content was $20 \%$ by volume. The CG was evaluated by the Fourier-Transformed Infrared spectroscopy method (FTIR). Results: There was an increase in DC for all groups as a function of time, with no statistical difference up to 5 s. From 11 s, the non-functionalized DCPD group presented lower DC than the other groups $(p<0.05)$. This behavior remained constant until the end of the evaluation. Conclusion: The functionalization of the DCPD nanoparticles statistically increased the degree of conversion of the experimental material.

DESCRIPTORS: Experimental composites; Degree of conversion; Orthodontic Materials

\footnotetext{
Aluno (a) do Curso de Graduação em Odontologia da Universidade UNG - Guarulhos- SP

Aluno (a) do Curso de Graduação em Odontologia da Universidade UNG - Guarulhos- SP

Aluna do Curso de Mestrado Profissional em Ortodontia da Universidade UNG - Guarulhos-SP

Aluna do Curso de Mestrado Acadêmico em Odontologia da Universidade UNG - Guarulhos-SP

4 Estatístico. Doutor em Estatística pelo Departamento de Medicina da Universitat de Barcelona. Professor dos Programas de Pós-Graduação Acadêmico em Odontologia e Mestrado Profissional em Ortodontia da Universidade UNG - Guarulhos-SP

5 Cirurgiã-Dentista. Doutora em Biomateriais e Biologia Oral pela Universidade de São Paulo. Professora do Programa de Pós-Graduação da Universidade Cruzeiro do Sul (UNICSUL) - São Paulo- SP

6 Cirurgião-Dentista. Doutor em Materiais Dentários pela Universidade de São Paulo. Professor Titular do Departamento de Biomateriais e Biologia Oral da Faculdade de Odontologia da Universidade de São Paulo (FOUSP) - São Paulo-SP

7 Cirurgiã-Dentista. Doutora em Biomateriais e Biologia Oral pela Universidade de São Paulo. Professora dos Programas de Pós-Graduação Acadêmico em Odontologia e Mestrado Profissional em Ortodontia da Universidade UNG - Guarulhos-SP (marina.roscoe@prof.ung.br)
} 


\section{RESUMEN}

Objetivo: Investigar la influencia de la adición de partículas de fosfato de calcio en el grado de conversión (GC) de los compuestos experimentales. Método: Se evaluaron cuatro compuestos experimentales: G1- Solo matriz orgánica basada en metacrilato (MM), G2- Adición de partículas de vidrio de bario (2 $\mu \mathrm{m})$, G3- Adición de nanopartículas de dihidrato de fosfato dicálcico (DCPD) funcionalizadas, G4- Adición de nanopartículas DPCD funcionalizadas con TEDGMA.El contenido de carga inorgánica fue del $20 \%$ en volumen. El GC se evaluó mediante el método de espectroscopía infrarroja transformada de Fourier (FTIR). Resultados: Hubo un aumento en el GC para todos los grupos en función del tiempo, sin diferencias estadísticas hasta 5 segundos. A partir de los 11 segundos, el grupo de DCPD puro presentó un GC más bajo que los otros grupos $(p<0.05)$. Este comportamiento permaneció constante hasta el final de la evaluación. Conclusión: La funcionalización de las nanopartículasde DCPD aumentó estadísticamente el GC del material.

DESCRIPTORES: Compositos experimentales; Grado de conversión; Materiales de ortodoncia. 


\section{INTRODUÇÃO}

A colagem direta de bráquetes ortodônticos substituiu o antigo sistema de cimentação de bandas, diminuindo o tempo e o trabalho do procedimento. Apesar deste grande avanço, a aparelhagem ortodôntica atual ainda dificulta o uso de medidas de higiene oral convencionais ${ }^{1}$ resultando em acúmulo de placa ao redor da base dos bráquetes e, consequentemente, em desmineralização do esmalte ${ }^{1}$. Este processo de desmineralização é extremamente rápido e tem como característica inicial o aparecimento de lesões de mancha branca $23,4,5$.

A incidência de lesões de mancha branca em pacientes tratados com aparelhos fixos chega a ser de até $50 \%{ }^{6,7}$. Métodos tais como a melhora da higiene oral, a modificação da dieta (diminuição da ingestão de carboidratos), e o tratamento com flúor tópico podem diminuir ou prevenir o aparecimento destas lesões. No entanto, estes métodos necessitam da colaboração efetiva do paciente para que a história natural da doença seja interrompida ${ }^{8}$.

Com o objetivo de reduzir o aparecimento de áreas de desmineralização ao redor dos bráquetes sem a total dependência do paciente, autores têm investigado a associação de agentes antimicrobianos à materiais ortodônticos ${ }^{9,10,11}$. Além de agentes antimicrobianos, fosfatos de cálcio (fosfato de cálcio amorfo, ACP, fosfato dicálcico anidro, DCPA, ou fosfato tetracálcico, TTCP) também vêm sendo adicionados à matriz resinosa no intuito de reduzir o risco de desenvolvimento de lesões de cárie secundária e de remineralizar lesões iniciais ${ }^{12}$.

Resultados promissores em estudos in vitro têm sido observados ${ }^{13,14}$.Entretanto, a adição destas partículas de fosfatos de cálcio à matriz diminui a interação química entre as fases orgânica e inorgânica, o que resulta em severa redução da resistência à fratura do material 15,16. No entanto, ainda não foi investigada a qualidade da adesão deste material ao esmalte dentário.

O esmalte é um substrato com grande conteúdo mineral e morfologia homogênea, o que faz com que os procedimentos adesivos no esmalte sejam considerados muito efetivos. No entanto, a qualidade desta adesão é dependente do grau de conversão do compósito, o qual irá influenciar diretamente as suas propriedades mecânicas ${ }^{17,18,19,20}$. Dessa forma, parece oportuno investigar a influência da adição de partículas de fosfato di-cálcico di-hidratado (DCPD) na cinética de polimerização e grau de conversão de materiais experimentais para colagem ortodôntica.

O objetivo deste estudo foi avaliar o efeito da adi- ção de partículas de fosfato di-cálcico di-hidratado na cinética de polimerização e no grau de conversão de compósitos para colagem ortodôntica.

\section{MÉTODO}

Para a primeira etapa deste estudo foram sintetizados dois tipos de partículas de fosfato di-cálcico di-hidratado (DCPD, CaHPO4 -2H2O): partículas puras e partículas funcionalizadas com dimetacrilato de trietileno glicol (TEGDMA ou ácido 2-metil-2-propenóico).

\section{Síntese de partículas de fosfato di-cálcico di-hidrata- do (DCPD) puras}

As partículas foram sintetizadas através de um processo sol-gel em meio aquoso envolvendo a reação estequiométrica entre o fosfato de amônio, (NH4)2HPO4 (PM: 132,06g/mol, lote SLBG6817V: Sigma Aldirch- St Louis, EUA), e o nitrato de cálcio, $\mathrm{Ca}(\mathrm{NO} 3) 2 \cdot 4 \mathrm{H} 2 \mathrm{O}$ (PM: 236,15 g/mol, lote MKBK6090V: Sigma Aldrich - St Louis, EUA). Soluções dos dois reagentes com concentrações iguais $(0,2 \mathrm{~mol} / \mathrm{L}: 0,078 \mathrm{~mol}$ de reagente em aproximadamente $390 \mathrm{~mL}$ de água deionizada e destilada) foram preparadas em temperatura ambiente e sob agitação magnética. As massas dos reagentes foram definidas de modo que a síntese resultasse em aproximadamente $10 \mathrm{~g}$ de cada material.

\section{Síntese de partículas de fosfato di-cálcico di-hidrata- do (DCPD) funcionalizadas com TEGDMA}

Para a síntese das partículas funcionalizadas, foi adicionado à solução de fosfato de amônio $15,4 \mathrm{~mL}$ de TEGDMA (PM: $286 \mathrm{~g} / \mathrm{mol}$, densidade: 1,092 g/L, ESSTECH, Technology Inc., Essington, PA), mantendo-se a proporção de $0,75 \mathrm{~mol}$ de monômero funcionalizante para cada mol de fosfato de amônio (solução receptora na síntese). As duas sínteses de partículas foram submetidas ao mesmo processo de precipitação e lavagem, onde a solução de nitrato de cálcio foi gotejada com auxílio de uma bomba peristáltica $(9 \mathrm{~mL} / \mathrm{min})$ sobre a solução de fosfato de amônio. Terminado o gotejamento, a solução final foi mantida sob agitação constante por 3 horas. Em seguida a suspensão de partículas foi filtrada com auxílio de um funil cerâmico e papel filtro, acoplado a uma bomba à vácuo. O precipitado de partículas foi submetido a uma série de três ciclos de lavagem para a remoção dos subprodutos da reação (nitrato de amônio) 
e do excesso de TEGDMA, no caso do DCPD funcionalizado. As lavagens consistiram na adição água destilada ao precipitado (300 mL), e agitação da mistura sob agitação magnética por 3 minutos. Em seguida, a solução era levada novamente ao sistema de filtração, em que a água removida era desprezada e iniciava-se um novo ciclo de lavagem. Ao final do terceiro ciclo, o precipitado foi congelado em freezer $\left(-80^{\circ} \mathrm{C}\right)$ e liofilizado (Virtisan SP Industries Company, modelo Benchtop, Gardiner, NY, EUA) até a obtenção de um pó branco.

\section{Síntese dos compósitos experimentais para cola- gem ortodôntica}

Quatro compósitos experimentais foram avaliados. O grupo 1 foi constituído apenas por matriz orgânica à base de metacrilato. Para os demais grupos, o conteúdo de carga inorgânica total foi de $20 \%$ em volume. Para o grupo 2 adicionou-se partículas de vidro de bário silanizado $(2 \mu \mathrm{m})$, para o grupo 3 adicionou-se nanopartículas de di-hidrato de fosfato dicálcico (DCPD) não funcionalizadas, enquanto que para o grupo 4 adicionou-se nanopartículas de DPCD funcionalizadas com TEDGMA.

Análise da cinética de polimerização e do grau de conversão dos materiais sintetizados experimentalmente

Para a análise da cinética de polimerização e do grau de conversão foi utilizado o método de espectroscopia (infravermelho) transformada de Fourier (FTIR), com um espectrômetro (Bruker Tensor 27 FT-IR, Massachusetts, EUA) equipado com um dispositivo de refletância total atenuado (ATR), composto por um cristal de diamante. Os compósitos experimentais dos 4 grupos avaliados $(3 \mu \mathrm{g})$ foram acomodados diretamente no cristal de diamante e fotopolimerizados por 20 segundos utilizando fotopolimerizador (LED RADII; SDI, Bayswater, Austrália) com irradiância de $1200 \mathrm{~mW} / \mathrm{cm}^{2}$ ( $\mathrm{n}=$ 7), regularmente verificada com radiômetro (Demetron, Danbury, EUA). Uma varredura foi adquirida a cada 1 $s$ durante a fotoativação. A análise foi realizada a uma temperatura ambiente controlada de $23^{\circ} \mathrm{C}( \pm 2 \circ \mathrm{C})$. Por meio da mensuração do grau de conversão observa-se a porcentagem de grupos vinil que se transformaram em grupos alifáticos. A avaliação foi realizada comparando os comprimentos de onda das bandas do metacrilato não polimerizado $\mathrm{C}=\mathrm{C}$, em $1638 \mathrm{~cm}-1$, com a banda aromática $\mathrm{C}=\mathrm{C}$, em $1610 \mathrm{~cm}-1$, usada como um padrão interno. Assim, a proporção de ligações duplas de carbono que são convertidas em ligações simples determinou o grau de conversão (GC).

Para a análise dos resultados quanto à cinética de polimerização e grau de conversão (GC) foi utilizada análise de variância com medidas repetidas e teste post-hoc de Bonferroni para as comparações entre os grupos, ao nível de significância estatística de 5\%.

\section{RESULTADOS}

A análise do grau de conversão e da cinética de polimerização demostrou que houve diferença estatisticamente significativa quanto ao tempo e ao grupo avaliado $(p<0,05)$. Houve um aumento de GC para todos os grupos em função do tempo, sem diferença estatística até $5 s(p>0,05)$. A partir de 11 segundos, o grupo DCPD puro apresentou grau de conversão inferior aos demais grupos $(p<0,05)$. Os demais grupos apresentaram grau de conversão similar estatisticamente a partir do tempo 11 segundos e este comportamento manteve-se constante até o final da avaliação (60 segundos).

Figura 1 - Análise da cinética de polimerização e do grau de conversão dos compósitos experimentais avaliados em função do tempo (em segundos).

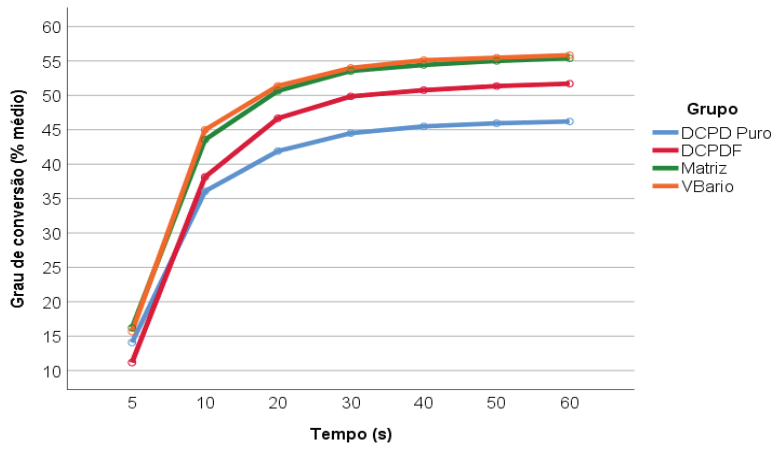

Tabela 1 - Análise da média do grau de conversão dos compósitos experimentais (em percentual) avaliados em função do tempo (em segundos).

Intervalo de tempo

Até $5 s \quad 6-10 s 11-20 s 21-30 s 31-40 s 41-50 s 51-60 s$

\begin{tabular}{cccccccc}
\hline DCPD Puro & $14,1 \mathrm{~A}$ & $36,1 \mathrm{~A}$ & $41,9 \mathrm{~A}$ & $44,5 \mathrm{~A}$ & $45,5 \mathrm{~A}$ & $45,9 \mathrm{~A}$ & $46,2 \mathrm{~A}$ \\
\hline DCPD F & $11,2 \mathrm{~A}$ & $38,1 \mathrm{AB}$ & $46,7 \mathrm{~B}$ & $49,9 \mathrm{~B}$ & $50,8 \mathrm{~B}$ & $51,3 \mathrm{~B}$ & $51,7 \mathrm{~B}$ \\
\hline Matriz & $16,2 \mathrm{~A}$ & $43,6 \mathrm{BC}$ & $50,7 \mathrm{~B}$ & $53,5 \mathrm{~B}$ & $54,4 \mathrm{~B}$ & $55,0 \mathrm{~B}$ & $55,4 \mathrm{~B}$ \\
\hline VBario & $15,7 \mathrm{~A}$ & $45,0 \mathrm{C}$ & $51,4 \mathrm{~B}$ & $54,0 \mathrm{~B}$ & $55,1 \mathrm{~B}$ & $55,5 \mathrm{~B}$ & $55,8 \mathrm{~B}$
\end{tabular}

Letras distintas indicam diferença estatisticamente significante $(p<0,05)$. 


\section{DISCUSSÃO}

É inegável que a possibilidade de realização da colagem diretados bráquetesortodônticos ao esmalte dentário contribuiu para a simplificação dosprotocolos de colagem e descolagem ${ }^{21}$ (Cardoso etal., 2014). Ainda assim, a presença de arcos, ligaduras e bráquetesortodônticos dificulta a higienização oral efetiva ${ }^{1,22}, 0$ que contribui para o acúmulo de biofilmecariogênico ao redor da base dos bráquetes e, consequente, desmineralização do esmalte'1.

Estudos in vitro e insitu têm mostrado resultados promissores avaliando materiais resinosos que incorporam partículas de fosfato de cálcio, no que diz respeito ao controle do desenvolvimento de lesões cariosas secundárias, por meio da remineralização ${ }^{13,14,23}$. Para que ocorra a remineralização, íons cálcio e fósforo devem ser liberados para a estrutura dentárias a partir de uma fonte externa. Estes íons depositam-se em espaços vazios do esmalte desmineralizado, acarretando em ganho mineral $^{24}$ (Cochraneetal., 2010). Para que este processo aconteça, deve haver dissolução e liberação de íons $\mathrm{Ca} 2+$ e HPO42- para o meio bucal, por meio do contato das diferentes fases de CaP com a saliva. Porém para que ocorra esta dissolução, é necessário que o CaP esteja subsaturado (em relação a concentração salivar) e que o $\mathrm{pH}$ bucal esteja abaixo do seu pH críti$\mathrm{co}^{24}$ (Cochraneetal.,2010).

Apesar dos resultados promissores em relação ao controle do desenvolvimento de lesões cariosas secundárias, a literatura científica já comprovou que a adição destas partículas de fosfatos de cálcio à matriz resinosa influencia negativamente as propriedades mecânicas do material, resultando em diminuição da resistência à fratura ${ }^{15,16}$. Este fato é decorrente da consequente di- minuição da interação química entre as fases orgânica e inorgânica quando partículas de fosfato de cálcio são adicionadas à matriz resinosa ${ }^{15,16}$.

No presente estudo foi observado que o grau de conversão de compósitos com a adição de nanopartículas de DCPD foi estatisticamente inferior em comparação aos compósitos experimentais sem adição de $20 \%$ de volume de DCPD. Esta fraca interação química entre as fases orgânica e inorgânica parece ser a responsável pela influência negativa da adição das partículas de DCPD à matriz resinosa, avaliando a cinética de polimerização e o grau de conversão destes materiais bioativos.

A funcionalização da superfície de partículas de fosfatos de cálcio com organosilanos tem sido investigada no intuito de melhorar a compatibilidade entre as partículas e a matriz resinosa e, consequentemente, melhorar as propriedades mecânicas do material ${ }^{25}$. O presente estudo demonstrou que a funcionalização das nanopartículas DCPD com TEGDMA aumentou de forma estatisticamente significante o grau de conversão do material experimental.

\section{CONCLUSÃO}

Os resultados obtidos no presente estudo indicaram benefício quanto a funcionalização das nanopartículas DCPD com TEGDMA. Contudo, trabalhos futuros devem ser realizados com o objetivo de sintetizar novos materiais com diferentes concentrações de DCPD em volume para identificar qual concentração seria capaz de controlar o desenvolvimento de lesões cariosas e promover a remineralização, mantendo as propriedades mecânicas dos compósitos resinosos utilizados em Ortodontia para a colagem de bráquetes. 


\section{REFERÊNCIAS}

1. Derks A, Katsaros C, Frencken JE, Van'tHof MA, Kuijpers-Jagtman AM. Caries- inhibiting effect of preventive measures during orthodontic treatment with fixed appliances. Caries Res. 2004 Sep-Oct; 38(5):413-20.

2. Gorelick L, Geiger AM, Gwinnett AJ. Incidence of white spot formation after bonding and banding. Am J Orthod. 1982;81:93-8.

3. Mizrahi E. Surface distribution of enamel opacities following orthodontic treatment. Am J Orthod 1983;84:323-31.

4. Årtun J, Brobakken BO. Prevalence of carious white spots after orthodontic treatment with multibonded appliances. Eur J Orthod. 1986;8:229-34.

5. Øgaard B, Rølla G, Arends J. Orthodontic appliances and enamel demineralization. Part 1. Lesiondevelopment. Am J Orthod Dentofacial Orthop. 1988;94:68-73.

6. Heintz S, Georg P. Oral health for the orthodontic patients. Philadelphia: Mosby; 1999. p. 2-9, 94-7.

7. Evrenol BI, Kucukkeles N, Arun T, Yarat A. Fluoride release capacities of four different orthodontic adhesives. J Clin Pediatr Dent. 1999;23:315-9.

8. Farhadian N, Miresmaeili A, Eslami B, Mehrabi S. Effect of fluoride varnishon enamel demineralization around brackets: anin-vivo study. Am J Orthod Dentofacial Orthop. 2008;133:S95-8.

9. Altmann AS, Collares FM, Leitune VC, Samuel SM. The effect of antimicrobial agents on bond strength of orthodontic adhesives: a meta-analysisofin vitro studies. Orthod Cranio fac Res. 2015 Aug10.

10. Jacobo C, Torrella F, Bravo-González LA, Ortiz AJ, Vicente $A$. In vitro study of the antibacterial properties and microbial. Colonization susceptibility of four self-etching adhesives used in orthodontics 2014;36:200-6.

11. Poosti M, Ramazanzadeh B, Zebar- jad M, Javadzadeh P, Naderinasab M, Shakeri MT. Shearbond strength and antibacterial effects of orthodontic composite containing TiO2 nanoparticles. Eur J Orthod. 2013;35:676-9.
12. Ferracane JL. Resin composite-state of the art. Dent Mater. 2011;27:29-38.

13. Langhorst SE, O'Donnell JN, Skrtic D. In vitro remineralization of enamel by polymeric amorphous calcium phosphate composite: quantitative microradiographic study. Dent Mater. 2009;25:884-91.

14. Weir MD, Chow LC, Xu HH. Remineralization of demineralized enamel via calcium phosphate nano composite. J Dent Res. 2012;91:979-84.

15. Xu HH, Weir MD, Sun L. Calcium and phosphate ion releasing composite: effectof $\mathrm{pH}$ on release and mechanical properties. Dent Mater. 2009;25:535-42.

16. Xu HH, Moreau JL. Dental glass-reinforced composite for caries inhibition: calcium phosphate ion release and mechanical properties. J Biomed Mater Res B Appl Biomater. 2010;92:332-40.

17. Bae JH, Cho BH, Kim JS, Kim MS, Lee IB, Son HH, Um CM, Kim CK, Kim OY. Adhesive layer properties as a determinant of dentin bond strength. J Biomed Mater Res B Appl Biomater. 2005 Aug;74(2):822-8.

18. Borges BC, Souza-Junior EJ, Brandt WC, Loguercio AD, Montes MA, Puppin- Rontani RM, Sinhoreti MA. Degree of conversion of simplified contemporary adhesive systems as influenced by extendedair-activatedor passive solvent volatilization modes. Oper Dent. 2012 May-Jun;37(3):246-52.

19. Argolo S, Mathias P, Aguiar T, Lima A, Santos S, Foxton R, Cavalcanti A. Effect of agitation and storage temperature on watersorption and solubility of adhesive systems. Dent Mater J.2015;34(1):1-6.

20. Hass V, Dobrovolski M, Zander-Grande C, Martins GC, Gordillo LA, Rodrigues AccorinteMde L, Gomes $\mathrm{OM}$, Loguercio AD, Reis A. Correlation between degree of conversion, resin-dentin bond strength and nanoleakage of simplified etch-and-rinse adhesives. Dent Mater. 2013 Sep;29(9):921-8. 
21.Cardoso LA, Valdrighi HC, Vedovello Filho M, Correr $A B$. Effect of adhesive remnant removal on enamel topography after bracket debonding. Dental Press J Orthod. 2014 Nov-Dec;19(6):105-12.

22. Ren $Y$, Jongsma MA, Mei L, van der Mei HC, Busscher HJ. Orthodontic treatment with fixed appliances and biofilm formation--a potential public health threat? Clin Oral Investig. 2014;18:1711-8.

23. Dickens SH, Flaim GM, Takagi S. Mechanical properties and biochemical activity of remineralizing resin-based Ca-PO4 cements. Dent Mater. 2003 Sep;19(6):558-66.
24. Cochrane NJ, Cai F, Huq NL, Burrow MF, Reynolds EC. New approaches to enhanced remineralization of tooth enamel. J Dent Res. 2010 Nov;89(11):1187-97.

25. Xu HH, Quinn JB. Whisker-reinforced bioactive composites containing calcium phosphate cement fillers: effects of filler ratio and surface treatments on mechanical properties. J Biomed Mater Res. 2001 Nov;57(2):165-74 